\title{
IDENTIFYING RECOMBINATIONAL LETHALS IN DROSOPHILA MELANOGASTER
}

\author{
J. L. BATTEN* + and J. M. THODAY \\ Department of Genetics, Milton Rood, Cambridge, England
}

Received 13.xi.68

\section{InTRODUCTION}

Since Dobzhansky (1946) first described the production of "synthetic" or " recombinational " lethals in $D$. pseudoobscura, many workers have investigated the phenomenon. In D. melanogaster, Misro (1949), Wallace, King, Madden, Kaufmann and McGunnigle (1953), Hildreth (1956) and Spiess and Allen (1961) all combined wild type chromosomes together in heterozygotes. Misro found that more lethals were produced from heterozygous females than from heterozygous males and attributed the excess to the formation of recombinational lethals. Similarly, Wallace et al. found that lethals were produced with greater frequency from heterozygous combinations, but Hildreth and Spiess and Allen failed to find any difference in the number of lethals produced by heterozygous and homozygous combinations. Gantner (1958), on the other hand, combined wild type chromosomes with a multiply marked laboratory chromosome and found no difference in the number of lethals in parental and recombinant type chromosomes obtained from such heterozygotes.

If two chromosomes can recombine to form one or more types of lethal chromosome, the frequency with which a particular lethal appears depends upon the distance separating the two regions or loci that when combined produce the lethal. If the components are situated far apart, the lethal should appear frequently, all such lethals should be allelic with each other, and the identification of recombinational lethals is easy, though there may be problems in the tests for allelism. Gibson and Thoday (1962), who discovered a recombinational lethal whilst investigating a selected line, synthesised their lethal many times and found that all the lethals thus produced were allelic.

In most investigations, however, the lethals have been less frequent and not allelic. Misro, for example, found all his lethals were non-allelic, a situation in which we face the major difficulty of this field of research, how to know unequivocally which of the lethals that turns up is mutational and which is recombinational in origin. Dobzhansky and Spassky (1960) tried to solve this problem by using desynthesis as a criterion of recombinational lethals. However, Thoday (1964) has pointed out that it may often be impossible to desynthesise a genuine recombinational lethal and it is clear that it may sometimes be possible to desynthesise a lethal of mutational origin.

Consider a recessive lethal in a gamete from a female heterozygous for a pair of "viable" chromosomes. That recessive lethal may have been

* Supported by a D.S.I.R. Research Studentship.

$\dagger$ Present address: Department of Genetics, University of Liverpool, Liverpool, England. 
mutational in origin, in which case we expect the origin of the lethality to have involved a single locus only, or it may be recombinational in origin, in which case its origin involved two loci. Operationally, however, once the lethal has occurred, distinction between the two modes of origin is impossible. As Thoday (1964) pointed out, if we map against a marked chromosome, the recombinational lethal will map at a single locus unless the marker happens to be of the reciprocal recombinant class (which in the case of Gibson and Thoday's (1962) lethal was impossible as the reciprocal recombinant was dominant lethal). Conversely, the mutational lethal will map at two loci if the marker happens to contain a suppressor at another locus, and then the mutational lethal will desynthesise and resynthesise just as if it were a recombinational lethal.

The distinction between mutational and recombinational lethals, can, therefore, only be made unequivocally if we use a technique similar to Gantner's (1958) in which we explore the production of lethals by chromosomes differing at marker loci that enable us to detect recombinants, or if we can demonstrate that a particular pair of chromosomes regularly produces the same lethal and that the same chromosomes do not do so when not allowed to recombine.

It follows that the recombinational lethal extensively studied by Gibson and Thoday is the only unequivocally recombinational lethal that has been at all fully studied. It seemed worthwhile, therefore, to seek to obtain further recombinational lethals by a technique that should permit us to know which of the lethals obtained are undoubtedly generated by recombination.

\section{MATERIALS AND METHODS}

Males, captured from several populations of $D$. melanogaster near Cambridge, England, in the summers of 1962 and 1963, were mated to $C y L^{4} / P m$ females and single male offspring were backcrossed to $C y L^{4} / P m$ females to extract individual II chromosomes from the natural populations. These were preserved in the laboratory as $C y L^{4}$ heterozygotes. All matings were made in either $3 \times 1$ in. vials or $\frac{1}{2}$-pint milk bottles containing standard oatmeal treacle agar medium and the cultures were reared at $25^{\circ} \pm 1^{\circ} \mathrm{C}$.

These extracted chromosomes were screened for their capacity to produce recessive lethals when heterozygous with a standard marked chromosome. $C y L^{4}$ males were mated to $d p b c n c b w$ females to produce several females heterozygous for the extracted chromosome and the "standard" $d p b c n c b w$ chromosome. These heterozygous females were mated to $C y L^{4} / P m$ males and 100 , or in some cases $300, C y L^{4}$ male offspring were made homozygous for their maternal II chromosomes by mating them to $C y L^{4} / P m$ females and mating the $C y L^{4}$ offspring together, see fig. 1. Those that were homozygous viable were discarded but those that failed to produce any non-Cy $L^{4}$ flies in any of four $3 \times 1$ in. vials, i.e. about 150 flies, were considered to be recessive lethal, were retained as $C y L^{4}$ heterozygotes and were mated to $d p b c n c b w$ females in order to determine the marker genotype of the maternal II chromosome.

Tests for allelism were performed on various combinations of these lethals and, in certain instances, the lethal was also mapped by mating males heterozygous for $C y L^{4}$ and the lethal to females homozygous for the $d p, b$, $c n, c$ and $b w$ marker genes not carried by the lethal chromosome. From the 
offspring, females heterozygous for this reciprocal recombinant class of chromosome and the lethal chromosome were backcrossed to males heterozygous for $C y L^{4}$ and the lethal chromosome. The non-lethal recombinants

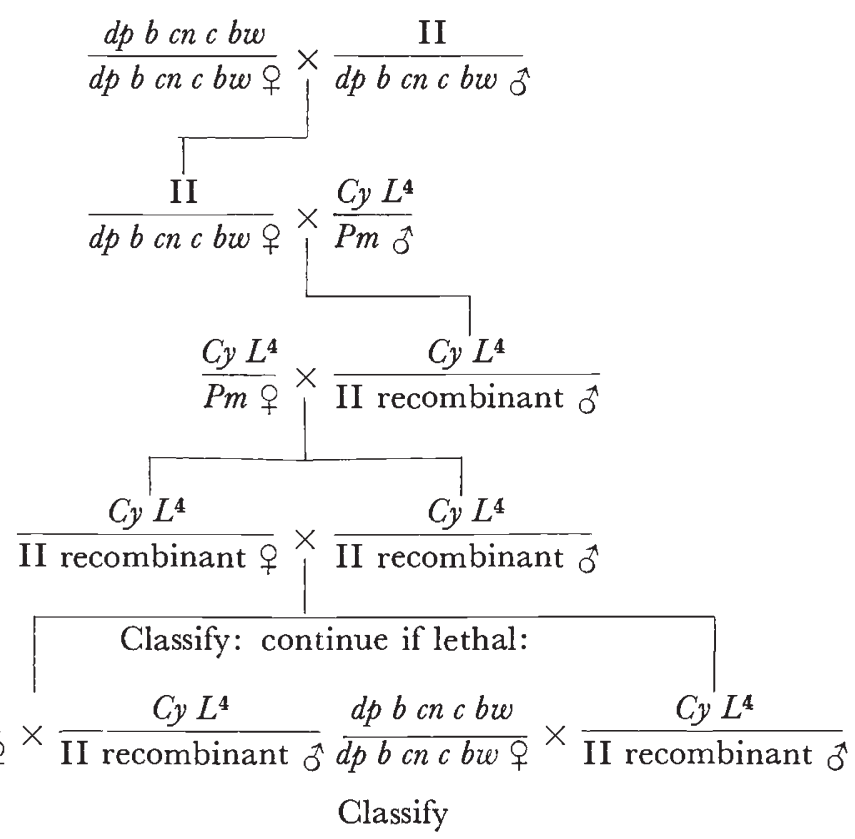

Classify

Classify

Fig. 1.--Breeding programme to determine the capacity of extracted II chromosomes to produce recessive lethals when heterozygous with a standard marker chromosome.

are heterozygous for the lethal chromosome and for non-lethal recombinants between this and the reciprocal class chromosome and were mated to $d p b$ cn $c$ bw females to identify their genotype; see fig. 2 .

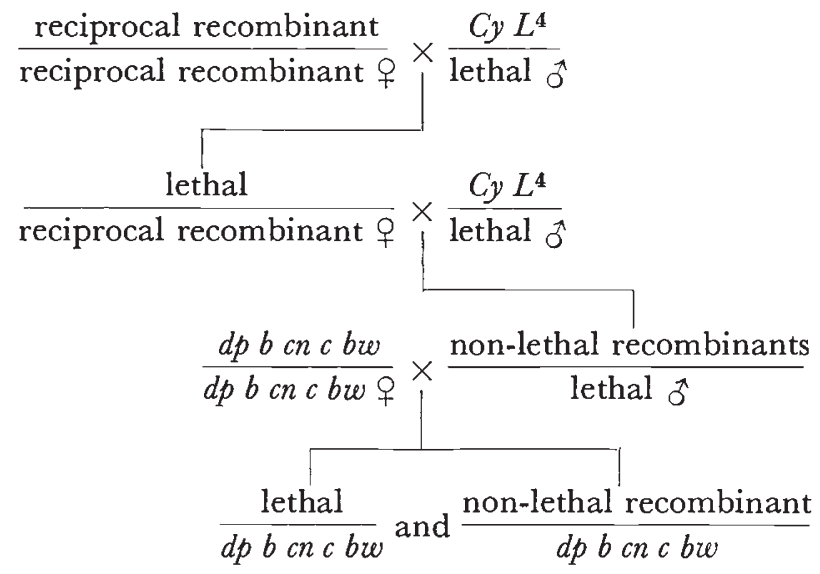

FIG. 2.-Breeding programme for the mapping of lethals from lethal recombinants. The reciprocal recombinant is a chromosome carrying the $d p, b, c n, c$ and $b w$ marker genes not carried by the lethal chromosome being mapped. 
Four types of lethal can be expected from the original breeding programme:

(a) Lethals that pre-existed or have arisen in the II or $d p b c n c b w$ chromosomes of the heterozygous female. These we call systemic lethals.

(b) Lethals that have arisen by mutation during the lifetime of the heterozygous female prior to meiosis. These we call premeiotic lethals.

(c) Lethals that have arisen by mutation in the egg cells of the heterozygous female. These are mutational lethals.

(d) Lethals that have arisen by recombination between the II chromosomes of the heterozygous female. These are recombinational lethals.

Table 1 shows how the tests for allelism and the location of the lethals will distinguish between these four types.

TABLE 1

The differences between the four types of lethal expected from the breeding programme of fig. 1

Origin of lethal

(a) Systemic lethals.

(b) Premeiotic lethals.

(c) Mutational lethals.

(d) Recombinational lethals.

\section{Parental and recombinant classes affected}

(a) One parental and half of the recombinant classes from individual heterozygous females.

(b) A fraction of one parental and half of the recombinant classes from individual heterozygous females.

(c) and $(d)$ A fraction of any one or more parental or recombinant classes from all females with the same heterozygous combination of chromosomes.

Tests for allelism

(a) and (b) Allelic.

(c) Not allelic.

(d) Allelic, provided that recombinational lethals are from the same loci.

Location of lethal

$(a),(b)$ and $(c)$ will map independently of the recombinant class in which it occurs.

(d) Will map in one or both of the regions corresponding to the recombinant class or classes in which it occurs.

\section{Results}

II chromosomes from 28 wild flies were passed through the initial breeding programme and table 2 gives the number and class of lethals obtained from 3553 of these resulting chromosomes. Three wild flies, $H B, L D$ and $L F$, contained recessive lethals and tests for allelism showed that all of their recessive lethal offspring were lethal in combination with the corresponding lethal parental chromosome, so that these lethals were classed as systemic.

Tests for allelism were carried out between the remaining 56 lethals. Only two combinations were found to be lethal: from $\mathrm{CH}$ a +++++ and $\mathrm{a}++++b w$ chromosome and, from $T L$, two $+b c n c b w$ chromosomes were lethal in combination with each other. The two lethal chromosomes from $\mathrm{CH}$ were both produced from the same female and were probably premeiotic. However, from $T L$, a further 950 chromosomes were obtained using the same breeding programme. From these, $16+b c n c b w$ and 5 $++c n c b w$ chromosomes were lethal. To confirm this, several females 


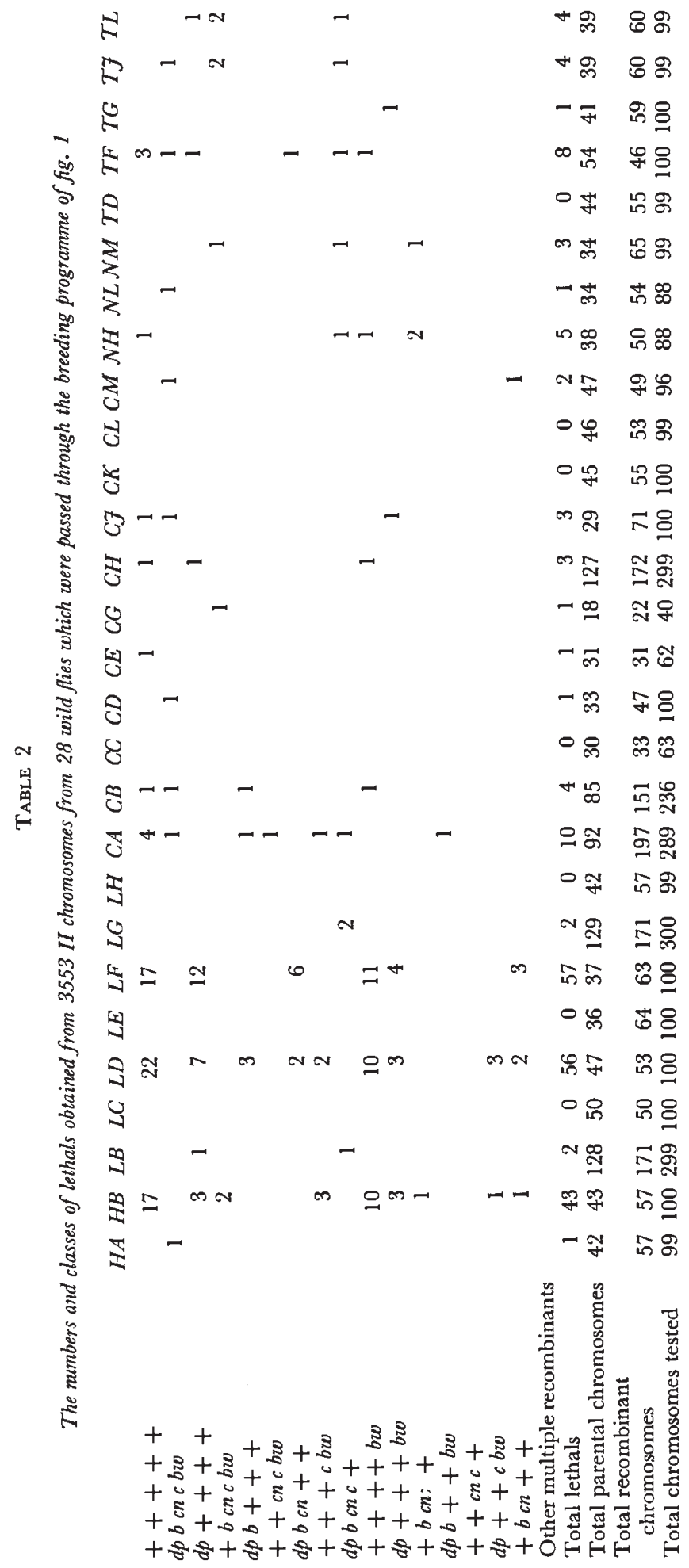


heterozygous for the $T L$ chromosome and $d p b c n c b w$ were backcrossed to $d p b c n c b w$ males and $160+b c n c b w$ and $40++c n c b w$ males were made homozygous using the $C y L^{4} / P m$ technique. The number of lethals and viables for both recombinant classes obtained by both methods are tabulated in table 3 and are in agreement with each other, $\chi^{2}<1 ; \mathrm{P}>0.05$ for both classes. Such lethals are likely to have been produced by a recombination between a pair of loci located between $d p$ and $b$ and $b$ and $c n$ at 41 and 52 respectively.

Tests for allelism between certain combinations of all these lethals were carried out. However, whereas most combinations produced no non-Cy $L^{4}$ flies, indicating that the lethals were allelic, about one quarter of the combinations produced non-Cy $L^{4}$ flies in numbers varying from between 1 per cent.

TABle 3

Lethals in the $+\mathrm{b} \mathrm{cn} \mathrm{c} \mathrm{bw}$ and $++\mathrm{cn} \mathrm{c}$ bw recombinant classes from females heterozygous for the TL and $\mathrm{dp} \mathrm{b} \mathrm{cn} \mathrm{c}$ bw chromosomes produced by (a) the breeding programme of fig. 1 and (b) backcrossing such females to males homozygous for the $\mathrm{dp} \mathrm{b} \mathrm{cn} \mathrm{c}$ bw chromosome

\begin{tabular}{|c|c|c|c|c|}
\hline Class & & Programme (a) & Programme (b) & Total \\
\hline$+b c n c b w$ & $\left\{\begin{array}{l}\text { Lethal } \\
\text { Viable } \\
\text { Total }\end{array}\right.$ & $\begin{array}{l}16 \\
60 \\
76\end{array}$ & $\begin{array}{r}27 \\
104 \\
131\end{array}$ & $\begin{array}{r}43 \\
164 \\
207\end{array}$ \\
\hline$++c n c b w$ & $\begin{array}{l}\text { Lethal } \\
\text { Viable } \\
\text { Total }\end{array}$ & $\begin{array}{r}5 \\
6 \\
11\end{array}$ & $\begin{array}{l}13 \\
20 \\
33\end{array}$ & $\begin{array}{l}18 \\
26 \\
44\end{array}$ \\
\hline
\end{tabular}

to 10 per cent. of the total flies present. Since such combinations were between pairs of lethals that were both lethal in combination with a third, it seemed likely that all the lethals were allelic, but that the lethal is " conditional " and that all the subviable heterozygotes were partly viable owing to their being heterozygous for genes on the $\mathrm{X}$ and III chromosomes. For certain of these combinations, males heterozygous for $P m$ and one lethal were mated to females heterozygous for $C y L^{4}$ and the other lethal. This mating was repeated for ten generations to produce both lethals on the same genetic background. The number of non-Cy $L^{4}$ heterozygotes was correspondingly decreased, which was to be expected on the view that the heterozygous genetic background was responsible for the appearance of the subviables.

Some evidence that the lethal $+b c n c b w$ and $++c n c b w$ chromosomes were obtained by recombination was provided by locating the lethal. Each of eleven of the original $C y L^{4} /+b c n c b w$ lethals were mated to $d p++++1 d p++++$ females to produce $d p++++1+b$ cn $c$ bw females which were mated to the corresponding lethal $C y L^{4} /+b c n c b w$ male. Thirty males of the non-lethal recombinant/lethal heterozygotes from each mating were progeny tested for the presence or absence of $d p$. For all eleven lethals, the $+++++,+b c n c b w, d p++++$, $d p+++b w, d p++c b w$ and $d p+c n c b w$ classes predominated among the offspring indicating that the lethals map between $d p$ and $b$. Similarly, each of four of the original $C y L^{4} /++c n c b w$ lethals were mated to $d p b+++$ homozygotes to produce $d p b+++1++c n c b w$ females which were mated to the corresponding lethal $C y L^{4} /++c n c b w$ male. 
Thirty males of the non-lethal recombinant/lethal heterozygotes from each mating were progeny tested for the presence or absence of $d p$ and $b$. For all four lethals, the $+b+++, d p+c n c b w, d p b+++, d p b++b w$, $d p b+c b w$ and $d p b c n c b w$ classes predominated among the offspring, indicating again that the lethal maps between $d p$ and $b$.

For the mapping of both the loci involved, and for the desynthesis of the lethal, the reciprocal recombinant chromosome had to be obtained. To do

$$
\begin{aligned}
& \frac{+-+++++}{d p+b-c n c b w \text { 우 }} \times \frac{C y L^{4}}{+-b-c n c b w \delta} \\
& \frac{+-+++++\mathrm{oR} d p \pm+++++}{+-b-c n c b w o} \times \frac{d p+b-c n+b w}{d p+b-c n+b w \delta} \\
& \frac{C y L^{4}}{+-b-c n c b w \text { q }} \times \frac{d p \pm b-c n c b w}{d p+b-c n+b w \sigma^{*}}
\end{aligned}
$$

Classify :

$$
\begin{aligned}
& \frac{1}{2} C y L^{4} \\
& \frac{1}{4}+b c n+b w
\end{aligned}
$$$$
\frac{1}{4}+b c n c b w \text {, if }+- \text { and not }--
$$

$+-+++++=$ original TL chromosome

$d p+b-c n c b w=$ standard marker chromosome

$d p+b-c n+b w=$ standard $d p b c n b w$ stock

$+-b-c n c b w=$ lethal chromosome

$d p \pm+++++=$ recombinant class which should contain some reciprocal recombinants to the lethal chromosome

$d p \pm b-c n c b w=$ recombinant class which should not contain any lethals if $d p++++$ chromosome is the reciprocal recombinant

FIG. 3.-The isolation of chromosomes which could be reciprocal recombinants of the lethal $+b c n c b w$ chromosomes.

this, females heterozygous for $d p b c n c b w$ and the original wild type chromosome capable of producing the recombinational lethal were mated to Cy $L^{4} /+b$ cn $c$ bw (lethal) males, and from these matings, 100 wild type female progeny were mated to $d p b c n+b w / d p b c n+b w$ males, and the 72 cultures containing no $d p / d p$ flies in the progeny were discarded. From each of the 28 remaining cultures, $d p++++$ males were preserved by mating to Cy $L^{4} / P m$ females and all the $d p b c n c b w$ males were mated to $C y L^{4} /$ $+b$ cn $c b w$ (lethal) females. The progeny from the latter set of cultures was classified for the presence of $+b c n c b w$ flies, for these could only have been produced if the male parental $d p b c n c b w$ chromosome was not lethal, and non-lethal $d p b c n c b w$ chromosomes could only have been produced from $d p++++$ females which carried the reciprocal recombinant to the lethal, see fig. 3, where - and - have been used to represent the alleles 
of the loci that have recombined to produce the recessive lethal, and + and + have been used to represent the other alleles at these loci. $d p b c n c b w$ progeny clearly viable in combination with the lethal $C y L^{\mathbf{4}} /+b c n c b w$

(1)

$$
\begin{aligned}
& \frac{C y L^{4}}{d p++++++q} \times \frac{C y L^{4}}{+-b-c n c b w \sigma^{\pi}} \\
& \frac{d p++++++}{+-b-c n c b w+9} \times \frac{d p+b-c n+b w}{d p+b-c n+b w \sigma} \\
& \frac{C y L^{4}}{P m+} \times \frac{d p \pm b-c n c b w}{d p+b-c n+b w \sigma} \quad \frac{C y L^{4}}{P m q} \times \frac{+-b \pm+++}{d p+b-c n+b w \delta^{\pi}}
\end{aligned}
$$

(2)

$$
\frac{C y L^{4}}{P m \text { ? }}
$$

$$
\begin{aligned}
& \frac{d p b c n c b w}{d p b c n c b w+} \times \frac{C y L^{4}}{d p \pm b-c n c b w} \\
& d p+b-c n+b w \delta
\end{aligned}
$$

Classify cultures (2) for $d p b c n c b w / d p b c n c b w$ and hence select from corresponding cultures (1):

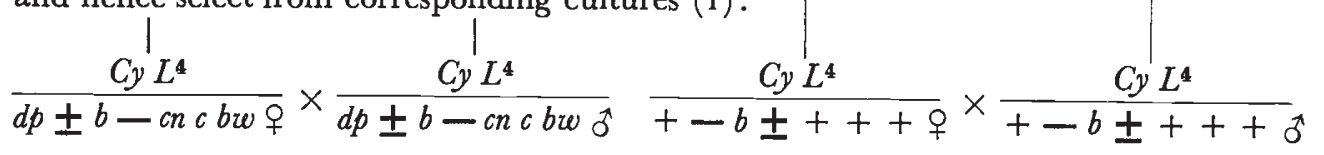

Classify:

$\frac{2}{8} C y L^{4}$

$\frac{1}{3} d p b c n c b w$, if +- and not - -
Classify:

$$
\frac{2}{3} C y L^{4}
$$$$
\frac{1}{3} d p b c n c b w \text {, if }-+ \text { and }
$$

$$
\text { not }--
$$

$d p++++++=$ reciprocal recombinant chromosome

$+-b-c n c b w \quad=$ lethal chromosome

$d p+b-c n+b w=$ standard $d p b c n b w$ stock

$d p b c n c b w \quad=$ standard marker stock

$\left.\begin{array}{l}d p \pm b-c n c b w \\ +-b \pm+++\end{array}\right\}=$ chromosomes which, if not lethal, are the products of desynthesis

FIG. 4:-The mapping of both loci involved and the desynthesis of the lethal $+b c n c b w$ chromosomes.

chromosome were obtained from five of the $28 d p++++$ recombinants. These five chromosomes are therefore of the required reciprocal recombinant class.

Fig. 4 shows how both loci were mapped. The reciprocal recombinant chromosome was combined with the recombinational lethal in heterozygous females which were mated to $d p b c n+b w / d p b c n+b w$ males. The ratio of lethal : viable $d p b$ cn $c b w$ recombinants was used to estimate the position 
of the left-hand locus in the $d p-b$ region and the ratio of lethal : viable $+b+++$ recombinants was used to estimate the position of the right-hand locus in the $b-c n$ region. Since the tests for allelism between the lethals had not been entirely satisfactory, the recombinants were tested for lethality by making them homozygous and not by testing them against a known lethal recombinant. Table 4 gives the results from 40 females, and these suggest that the left-hand locus maps at 40 and the right-hand locus at 52. These estimates assume that all the lethals are recombinational. If one or more of the lethals are actually mutational, the true positions will lie to the left.

TABLE 4

Results of lethal tests on the $+\mathrm{b}+++$ and $\mathrm{dp} \mathrm{b} \mathrm{cn} \mathrm{c}$ bw recombinants from 40 females which were heterozygous for the reciprocal recombinant chromosome and the recombinational lethal chromosome

$\begin{array}{ccccc} & \text { Viable } & \text { Lethal } & \text { Total } & \% \text { Viable } \\ d p b \text { c } c b w & 41 & 139 & 180 & 22 \cdot 8 \\ +b+++ & 23 & 29 & 52 & 44 \cdot 3\end{array}$

The breeding programme in fig. 4 not only permits mapping of the two loci involved, but also involves desynthesis of the lethal: the viable $d p b c n c b w$ and $+b+++$ are the desynthesised products. This was confirmed by combining the $+b+++$ and $+b c n c b w$ chromosomes and mating such a heterozygous female to $d p b c n c b w / d p b c n c b w$ males. From the offspring, $30+b c n c b w$ males were made homozygous and five of these were found to be homozygous lethal, showing that the lethal could be resynthesised again.

\section{Discussion}

From 28 wild second chromosomes in combination with a standard marker, only one definite recombinational lethal system was found. If wild populations of Drosophila are heterozygous at many of their loci, more recombinational lethals might be expected than have been found in this investigation. It is possible that the populations sampled were established by only one or two flies, homozygous at most of their loci, but selection experiments such as those of Thoday (Thoday and Boam, 1959; Thoday and Gibson, 1962) show that not all British populations of Drosophila have a low genetic variability and would, in fact, suggest the contrary.

It is also possible that the loci capable of producing recombinational lethals may usually be located close together as is indicated by the frequency of recombinational lethals found by other workers. Such lethals would not be detected as recombinational when only 100 or 300 chromosomes from a female heterozygote are made homozygous, for as explained in the introduction, a lethal must be produced more than once if it is to be shown to be recombinational in origin. However, from the 25 chromosomes that were homozygous viable, omitting the two premeiotic and the two recombinational lethals, only 52 lethals were obtained from 3249 derived chromosomes, which is 1.6 per cent., and could easily be obtained from mutation alone, see Plough (1941), Dubinin (1946), Misro (1949), Ives (1945, 1950), and Spiess and Allen (1961). Further, this proportion is equally distributed through the parental and recombinant classes which also suggests that the 52 lethals are mutational in origin. 
It was fortunate that the recombinational lethal investigated had its components located each side of a marker gene. Desynthesis of a recombinational lethal which lies within a chromosome marker segment is possible, but requires large numbers of cultures, firstly to ensure the generation of the reciprocal recombinant, and secondly to distinguish the desynthesised chromosomes which can resynthesise the recombinational lethal, from the reciprocal recombinant chromosome. Dobzhansky and Spassky (1960) were fortunate that the two loci affecting the lethal they desynthesised were at opposite ends of the chromosome. On the other hand, the reciprocal recombinant to the recombinational lethal discovered by Gibson and Thoday (1962) was dominant lethal, so that desynthesis of their lethal was not possible.

\section{TABLE 5}

A comparison of the number of lethals produced from heterozygous females and males, homo zygous females and inversion hetero zygotes. Only the wild-type products were tested from the inversion heterozygotes

\begin{tabular}{|c|c|c|c|c|}
\hline Parental genotype & Chromosomes & ested & Lethal & $\%$ lethal \\
\hline$\frac{T L}{d p b c n c b w \text { + }}$ & & 917 & 39 & $4 \cdot 3$ \\
\hline & $\int^{T L}$ & 317 & 2 & $0 \cdot 6$ \\
\hline$\frac{T L}{d p b c n c b w \sigma^{\pi}}$ & $d p b c n c b w$ & 155 & 3 & 1.9 \\
\hline & Total & 472 & 5 & $1 \cdot 1$ \\
\hline$\frac{1 L}{T L Q}$ & & 398 & 15 & $3 \cdot 3$ \\
\hline$\frac{d p b c n c b w}{d p b c n c b w \text { + }}$ & & 467 & 10 & $2 \cdot 1$ \\
\hline$\frac{C y L^{4}}{T L q}$ & & 476 & 14 & $3 \cdot 6$ \\
\hline
\end{tabular}

The phenomenon of heterozygous combinations of similar lethals being sub-lethal has been noted by Goldschmidt, Wahrmann, Ledermann-Klein, and Weiss (1955) and has several unfortunate consequences. It is not always possible to confirm that lethals produced from different wild chromosomes are allelic without several generations of inbreeding. Secondly, it prevents the use of a recombinational lethal to select against similar lethals and finally, it may interfere with attempts to distinguish between mutational and recombinational lethals by desynthesis, for recombinations may produce a new heterozygous chromosome segment which causes the lethal in effect to appear as viable by acting as a suppressor to the lethal. Such a possibility was reduced in the breeding programme of fig. 4, since the bw locus of the $d p b c n+b w$ chromosome could enable recombination in the right arm to be recognised.

The method for identification of recombinational or synthetic lethals we have used here clearly demonstrates that such lethals occur. The results also show that any one wild chromosome must be investigated on a large scale if the repeated production of the same lethal by recombination in the same region of the linkage map is to be demonstrated.

As we have argued above, this criterion is the only one which will unequivocally demonstrate the recombinational origin of a lethal. In this connection we would point out that the classical evidence that synthetic 
lethals occur as used for example by Misro (1949), who showed that the same pair of chromosomes combined in females produced more lethal gametes than when combined in males, though highly suggestive, is inadequate, for it is based upon the assumption that tested chromosomes mutate as frequently in males as in females.

We have specifically tested this point, using the wild chromosome that we have shown to be capable of producing a lethal by recombination with $d p b c n c b w$ by suppressing recombination in females in different ways. The results are given in table 5 , in which it is clear that the homozygous females and the inversion heterozygotes produce a higher frequency of lethals than the heterozygous males.

\section{Summary}

1. Twenty-eight II chromosomes extracted from natural populations of Drosophila melanogaster were tested for their ability to form lethals by recombination with a non-lethal marked chromosome.

2. Satisfactory evidence that recombination could produce lethals was only obtained with one chromosome, and the lethal this produced was intensively studied. The reciprocal recombinant was identified, the genes responsible were located, and the lethal was "desynthesised" into its component parts and "resynthesised".

\section{REFERENCES}

DOBZhansky, тн. 1946. Genetics of natural populations. XIII. Recombination and variability in populations of $D$. pseudoobscura. Genetics, 31, 269-290.

DOBZHANSKY, TH. AND SPASSKY, B. 1960. Release of genetic variability through recombination. V. Breakup of synthetic lethals by crossing over in Drosophila pseudoobscura. Zool. Jarhb., 88, 57-66.

DUbinin, N. P. 1946. On lethal mutations in natural populations. Genetics, 31, 21-38.

GANTNER, J. F. 1958. The frequency of lethals in crossover and non-crossover second chromosomes of $D$. melanogaster. Genetics, 43, 448-457.

Gibson, J. B., AND THODAY, J. M. 1962. Effects of disruptive selection. VI. Analysis of a second chromosome polymorphism. Heredity, 17, 1-26.

GOLDSCHMIDT, E., WAHRMANN, J., LEDERMANN-KLEIN, A., AND WEISS, R. 1955. A two year's survey of population dynamics in Drosophila melanogaster. Evolution, 9, 353-366.

HILDRETH, P. E. 1956. The problem of synthetic lethals in D. melanogaster. Genetics, 41, 729 742.

IVES, P. T. 1945. The genetic structure of American populations of Drosophila melanogaster. Genetics, 30, 167-196.

IVES, P. T. 1950. The importance of mutation rate genes in evolution. Evolution, 4, 236-252. MISRO, B. 1949. Crossing over as a source of new variation. Proc. 8th Intern. Congr. Genet., 629-630.

PLOUGH, H. H. 1941. Spontaneous mutability in Drosophila. C.S.H.S.Q.B., 9, 127-137.

SPIESS, E. B., AND ALLEN, A. C. 1961. Release of genetic variability through recombination.

VII. Second and third chromosomes of Drosophila melanogaster. Genetics, 46, 1531-1533. thoday, J. м. 1964. Locating synthetic lethals. Amer. Nat., 97, 353.

THODAy, J. M., AND BOAM, т. в. 1959. Effects of disruptive selection. II. Polymorphism and divergence without isolation. Heredity, 13, 205-218.

THODAY, J. M., AND GIBSON, J. B. 1962. Isolation by disruptive selection. Nature, 193, $1164-1166$.

WAllace, B., King, J. C., madden, C. v., KaUfmann, B., And mCGunnigle, E. C. 1953. An analysis of variability arising through recombination. Genetics, 38, 272-307. 